\title{
Application of a Phase-Field Method to the Numerical Analysis of Motions of a Two-phase Fluid with High Density Ratio on a Solid Surface"
}

\author{
Naoki TAKADA ${ }^{* *}$, Junichi MATSUMOTO**, Sohei MATSUMOTO** \\ and Naoki ICHIKAWA ** \\ ${ }^{* *}$ Advanced Manufacturing Research Institute, National Institute of Advanced Industrial Science and \\ Technology (AIST), \\ 1-2-1 Namiki, Tsukuba-shi, Ibaraki 305-8564, Japan \\ E-mail: naoki-takada@aist.go.jp
}

\begin{abstract}
A numerical method for solving Navier-Stokes equations and combined with a phase-field interface model is applied to flow problems of motion of an incompressible isothermal two-phase fluid with a high density ratio on a solid surface. Based on the free-energy theory, a fluid interface is described as a finite volumetric zone across which the physical properties vary continuously. The wettability of a solid surface is taken into account through a simple boundary condition derived from the increase in free energy on the surface. The phase-field approach simplifies the capture of motions of a fluid interface on a surface (contact line). The major findings from the simulations are as follows: (1) the contact-line motions of the liquid column under gravity are well predicted in comparison with the available data; (2) the static contact angle is flexibly controlled by a parameter of the wetting potential of the surface; (3) the capillary force is evaluated appropriately; (4) the acceleration of the two-phase flow in a channel with a local hydrophilic surface is predicted and observed to be in qualitative agreement with the experimental data; and (5) the displacement and breakup of a single drop on a flat solid wall are well predicted qualitatively. These results prove that the phase-field method can be employed for simulating air-water flows on a surface with heterogeneous wettability.
\end{abstract}

Key words: Computational Fluid Dynamics, Diffuse-Interface Model, Interface Tracking, Wettability, Contact Line

\section{Introduction}

Two-phase flows of a fluid-fluid interface moving on a solid surface are widely encountered in various fields of science and engineering. On a surface under hydrodynamic non-slip conditions, the velocity of the interface differs from that of the viscous fluid. For instance, the flow velocity must be zero on a stationary surface, whereas the fluid-fluid interface, which is the so-called contact line, may be moved on the surface. The wettability of the surface may affect the two-phase flow of the contact lines, particularly in situations in which the surface tension force is dominant over other forces ${ }^{(1)-(3)}$. In cases of heterogeneous wettability, the contact angle between the interface and the surface varies with location. Such variation in the contact angle causes complicated motions of the fluid 
with large deformation of the contact line due to the difference in the capillary force. Computational fluid dynamics (CFD) simulations facilitate the understanding and prediction of such complex two-phase flow phenomena; as its result, fluidic devices can be optimized for flexible and accurate flow control. It is often difficult to experimentally observe such flows inside them in three dimensions or to analyze theoretically them by employing the classical continuum-dynamics approach by using a sharp-interface model due to the stress singularity at the contact line ${ }^{(4)-(6)}$. Therefore, it is important to develop CFD methods for analyzing the flow problems.

The purpose of this study is to examine the applicability of a CFD method ${ }^{(7)}$, one of phase-field methods (PFMs) ${ }^{(8),(9)}$, for the analysis of the motions of two-phase fluid with high density ratio on a solid surface. PFMs employed for CFD simulations introduce a mesoscopic interface model based on the free-energy theory ${ }^{(10),(11)}$ into the framework of macroscopic governing equations of the flows based on one-field formulation. In PFM, an interface is described as a finite volumetric zone across which physical properties vary steeply but continuously. Such volumetric zones of the fluid are autonomously formed to retain its width by a diffusion current induced by the gradient of chemical potential, which minimizes the free energy of the fluid system and leads it toward an equilibrium state through phase separation or phase change. Such interfacial zones are discretized with several mesh units on a computational grid with sufficient spatial resolution to evaluate the gradients of the properties. The surface tension of the interface is defined as the increase in energy per unit area due to the local gradient of a so-called order parameter that represents the properties (e.g., density or concentration) ${ }^{(9)}$. The effect of the increase in energy appears as a body force distributed continuously only inside the interface. The contact angle, depending on the energy balance among three types of interfaces, is obtained from the wetting potential of the solid surface through a simple boundary condition of the gradient of the order parameter on the surface ${ }^{(4)-(6)}$. The diffuse-interface model simplifies the interface-tracking calculation by using standard finite difference techniques alone ${ }^{(8),(9)}$, and without employing any elaborating algorithms for the advection and reconstruction of interfaces and the evaluation of surface force in other methods ${ }^{(12)}$. Therefore, the PFM using the diffuse-interface model has attracted considerable attention from many researchers as a useful CFD tool for two-phase flow simulations ${ }^{(4)-(6),(8),(9),(13)-(15)}$.

Hereafter, a PFM that directly solves Navier-Stokes (NS) equations is called as NS-PFM. The PFM used in this study ${ }^{(7)}$ is equivalent to one of the two-phase lattice Boltzmann methods (LBMs) ${ }^{(3),(6),(16)-(20)}$, which is applicable to incompressible isothermal flows with high density ratio ${ }^{(18)}$. One of the advantages of the NS-PFM over LBM is that it saves computational memory because the number of macroscopic variables in NS equations is generally less than that of mesoscopic variables for the number density of fictitious fluid particles in LBM kinetic equations. Although both the NS-PFM and LBM have been successfully applied to various two-phase flow problems ${ }^{(3)-(6),(8),(9),(13)-(20)}$, the applications of the PFM to contact-line problems at high density ratios greater than 800 have been considerably less, except for the recent applications ${ }^{(21)}$.

In the next section, we briefly explain a NS-PFM ${ }^{(7)}$ and the wetting boundary condition on a solid surface ${ }^{(4)-(6)}$ in a two-phase flow with high density ratio. The third section shows the numerical results of the collapse of a liquid column under gravity, static contact angle, two-phase flows in a channel, and displacement and breakup of a single drop on a flat solid surface with heterogeneous wettability. The conclusion is described in the last section.

\section{Numerical Method Based on the Diffuse-Interface Model}

\subsection{Basic equations}

The phase-field method used in this study, NS-PFM, which is based on a LBM ${ }^{(18)}$, has been proposed for incompressible, isothermal gas-liquid flows with high density ratio ${ }^{(7)}$. 
The method solves a set of mass and momentum conservation equations of the fluid, and the advection-diffusion equation of the diffuse-interface profile ${ }^{(5),(14),(15)}$,

$$
\begin{aligned}
& \nabla \cdot \mathbf{u}=0, \\
& \frac{\partial \mathbf{u}}{\partial t}+\mathbf{u} \cdot \nabla \mathbf{u}=\frac{1}{\rho}\left[-\nabla \cdot \mathbf{P}+\nabla \cdot \mu\left(\nabla \mathbf{u}+\nabla \mathbf{u}^{T}\right)\right], \\
& \frac{\partial \phi}{\partial t}+\nabla \cdot(\phi \mathbf{u})=-\nabla \cdot[-\Gamma(\phi) \nabla \eta],
\end{aligned}
$$

where u denotes the velocity; $t$, the time; $\rho$, the density; $\mathbf{P}$, the pressure tensor; $\mu$, the viscosity; $\mathbf{g}$, the gravity; $\phi$, the order parameter ${ }^{(9)}$; and $\eta$, the chemical potential.

The scalar variable $\phi$ distinguishes between two phases with different values of $\phi$, and it indicates an interface as a finite volumetric zone between the phases across which $\phi$ varies continuously ${ }^{(5),(14)-(16)}$. Inside the interface, $\rho$ is assumed to be a sine-curve function of $\phi$ that varies between given constants for gas and liquid phases, $\rho_{G}$ and $\rho_{L} ; \mu$ varies between the given constants $\mu_{G}$ and $\mu_{L}$ as a linear function of $\rho^{(18)}$.

In this study, for two-phase flows with constant surface tension $\sigma, \mathbf{P}$ is given by the following expression ${ }^{(3),(14)}$, which is a simplified form of its full expression ${ }^{(8),(9)}$.

$$
\nabla \cdot \mathbf{P}=\nabla p-\rho \kappa_{S} \nabla\left(\nabla^{2} \rho\right)
$$

where $p$ denotes the pressure in a homogeneous field and it is regarded as a scalar variable. In this study, the parameter $\kappa_{S}$ is set to be a constant in the entire field; this is based on the following definition of $\sigma$ on a flat interface normal to the direction of axis $\xi^{(8),(9),(11)}$.

$$
\sigma \equiv \kappa_{S} \int_{-\infty}^{+\infty}\left(\nabla_{\xi} \rho\right)^{2} d \xi
$$

The chemical potential $\eta$ is derived from the bulk free energy $\psi$ and the excessive energy caused by the squared local gradient of $\phi^{(9),(11)}$.

$$
\eta \equiv \frac{\partial \psi}{\partial \phi}-\kappa_{\phi} \nabla^{2} \phi
$$

where $\psi$ represents the double-well form of $\phi^{(8),(9)}$ and $\kappa_{\phi}$ is a parameter for controlling the interfacial thickness ${ }^{(18)}$. In this study, the van-der-Waals free energy ${ }^{(10)}$ is represented by $\psi(\phi)^{(8),(17)-(20)}$. For simplicity of computation, the mobility $\Gamma(\phi)$ is defined as $\Gamma_{0} \phi^{(7),(18)}$, where $\Gamma_{0}$ is a given positive constant ${ }^{(7)}$. The right-hand side of Eq.(3) influences the formation of the interfaces with a given constant thickness, and this factor disappears in the equilibrium state.

By using the reference variables of velocity $u_{r}$, density $\rho_{r}$, viscosity $\mu_{r}$, order parameter $\phi_{r}$, and length $L_{r}$, Eqs.(2) and (3) are converted to non-dimensional equations, as follows (14),(15),(17):

$$
\begin{aligned}
& \frac{\partial \mathbf{u}}{\partial t}+\mathbf{u} \cdot \nabla \mathbf{u}=\frac{1}{\rho}\left[-\nabla p+\frac{1}{R e} \nabla \cdot \mu\left(\nabla \mathbf{u}+\nabla \mathbf{u}^{T}\right)\right]+\frac{1}{K}\left(\frac{\kappa_{S} \rho_{r}^{2}}{L_{r} \sigma}\right) \nabla\left(\nabla^{2} \rho\right) \\
& \frac{\partial \phi}{\partial t}+\nabla \cdot(\phi \mathbf{u})=\nabla \cdot\left(P e^{-1} \nabla \phi\right)
\end{aligned}
$$

In the above expressions, $t, \nabla, \mathbf{u}, p, \rho, \mu$, and $\phi$ have been normalized. The parameters $R e$ and $P e$ denote the Reynolds and Peclet numbers, respectively; $K$ is identified with either the capillary number $\mathrm{Ca}$ or the square of the Ohnesorge number $\mathrm{Oh}$ in each of the simulations mentioned below.

$$
R e=\frac{\rho_{r} L_{r} u_{r}}{\mu_{r}}, P e=L_{r} u_{r}\left(\Gamma_{0} \phi \frac{\delta \mu}{\delta \phi}\right)^{-1}, C a=\frac{\mu_{r} u_{r}}{\sigma}, O h=\frac{\mu_{r}}{\sqrt{\rho_{r} \sigma L_{r}}} .
$$

It must be noted that, in Eq.(8), the diffusion coefficient of $P e$ may assume a negative value to retain the profile of $\phi$ with a given constant width proportional to $\kappa_{\phi}^{0.5(7),(11)}$.

\subsection{Boundary condition on solid surface}

In a flow, a solid surface with velocity $\mathbf{u}_{\mathrm{w}}$ is regarded as a no-flux and no-slip boundary, 
on which the following constraints are imposed on the fluid motion ${ }^{(22)}$ :

$$
\begin{aligned}
& \mathbf{n} \cdot \nabla p=0, \\
& \mathbf{n} \cdot \nabla \eta=0, \\
& \mathbf{u}=\mathbf{u}_{W},
\end{aligned}
$$

where $\mathbf{n}$ is the unit vector normal to the boundary. In addition, for $\rho_{G} \leq \rho \leq \rho_{L}$, the following constraint of wettability is taken into account in a manner similar to that in other PFMs (3)-(6),(20).

$$
\mathbf{n} \cdot \kappa_{\phi} \nabla \phi=-\gamma_{S}
$$

The parameter $\gamma_{S}$ is called as the wetting potential ${ }^{(20)}$. The above equation is derived from a free-energy functional $\Psi_{\text {system }}$ of the entire fluid system with volume $V^{(4)-(6)}$.

$$
\Psi_{\text {system }} \equiv \int_{V}\left[\psi(\phi)+\frac{\kappa_{\phi}}{2}|\nabla \phi|^{2}\right] d V-\int_{S} \gamma_{S} \phi d S,
$$

where $-\gamma_{S} \phi$ denotes the increase in energy per unit area on the surface $S$, and $\gamma_{S}=0$ leads to a static contact angle $\theta_{W}=90^{\circ}$.

\subsection{Numerical scheme and algorithm}

Under the boundary condition given in Eqs.(10)-(13), Eqs.(1)-(3) are solved by using the following conventional techniques ${ }^{(7),(23),(24)}$. The three-dimensional space is discretized uniformly by using unit cubic cells on a fixed structured grid with mesh width $\Delta x=\Delta y=\Delta z$ $=1$ in the Cartesian coordinate system $(x, y, z)$, where the scalar and vector variables are located in a staggered arrangement. The gradients of $p, \phi, \eta$, and $\rho$ are evaluated with a fourth-order central difference scheme (CDS). The advancement in time $t$ is based on the second-order Runge-Kutta's scheme for constantly increasing $\Delta t$. The variables $p$ and $\mathbf{u}$ are obtained by using the projection method ${ }^{(25)}$. In Eq.(2), the advection is calculated with a third-order upwind finite difference scheme ${ }^{(26)}$, whereas the viscous stress is evaluated with a second-order CDS. In Eq.(3), both the terms of advection and diffusion of $\phi$ are calculated by using a fourth-order CDS within the framework of the finite volume method for volume conservation within the entire computational domain. On the solid boundary, $\phi$ is extrapolated by using the known data within the flow domain according to the wetting condition of Eq.(13), as follows:

$$
\begin{aligned}
& \phi_{-1}=\phi_{0}+\gamma_{S} \Delta x \kappa_{\phi}^{-1}, \\
& \phi_{-2}=\phi_{1}-27\left(\phi_{0}-\phi_{-1}\right)-24 \gamma_{S} \Delta x \kappa_{\phi}^{-1},
\end{aligned}
$$

where the subscript of $\phi$ denotes the cell number along the $x$ direction and normal to the solid surface and the number $=0$ indicates that the cell neighbors the surface inside the flow domain (see Fig.1). The equation (16) is given by using a finite difference approximation of $\partial \phi /\left.\partial x\right|_{-1 / 2} \approx\left\{-\phi_{1}+27\left(\phi_{0}-\phi_{-1}\right)+\phi_{-2}\right\} /(24 \Delta x)$.

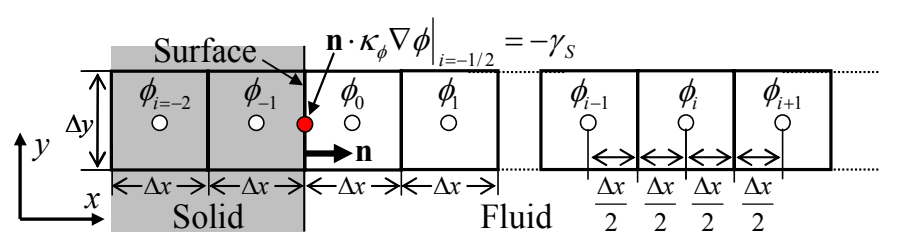

Fig. 1 Spatial arrangement of the scalar variable $\phi$ in the computational domain with solid boundary.

\section{Numerical Results and Discussion}

All the simulations were carried out under the condition that the gas-liquid density ratio $\rho_{L} / \rho_{G}$ was almost equivalent to that of the air-water system. The major parameters were as

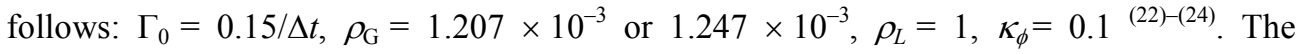
maximum and the minimum values of $\phi$ were 0.405 and 0.265 across a flat interface, respectively ${ }^{(7)}$. The transition width of $\phi$ was $8.96 \Delta x$, whereas that of $\rho$ was $3.96 \Delta x$, according to a linear approximation of the gradients. The diffusive interface was represented 
by a contour surface with medium value of the density $\left(\rho_{\mathrm{G}}+\rho_{L}\right) / 2$, when evaluating the interface position for comparison with the available data or visualization of the result. The parameter values shown are dimensionless.

\subsection{Motion of contact line on a neutrally wettable solid surface}

For examining contact line motion, the NS-PFM was first applied to the collapse of a two-dimensional liquid column in a gas under gravity $\mathbf{g}$ in a rectangular domain surrounded with neutrally wettable solid walls $\left(\theta_{W}=90^{\circ}\right)$. The simulation was conducted for aspect ratio of the column $n^{2}=H / a=1$ and 2 . In both the cases of $n^{2}$, the initial column width $a=$ 40 cells was assumed to be equivalent to 2.25 inches in the air-water system. The effect of $\mathbf{g}$ on the fluid was taken into account only in the regions with $\rho>\rho_{G}$.
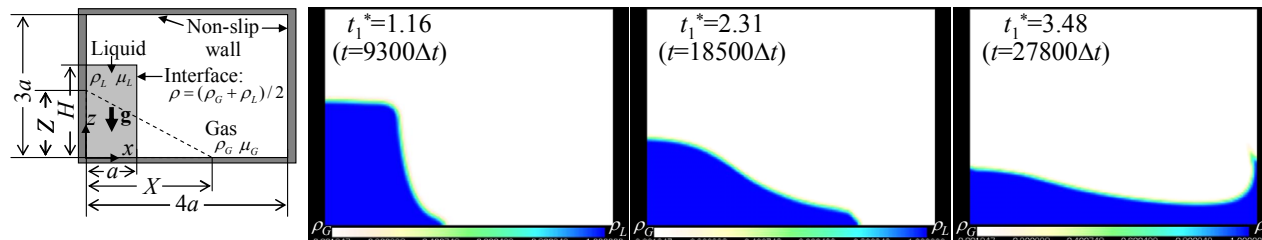

Fig. 2 Collapse of the two-dimensional liquid column under gravity in gas for aspect ratio $n^{2}=H / a=2$.

The time series variation in the shape of the column at $n^{2}=2$ is shown in Fig.2. The dimensionless time values $t_{1}{ }^{*}=n t(|\mathbf{g}| / a)^{0.5}=1.16,2.31$, and 3.48 correspond to $0.1,0.2$, and $0.3 \mathrm{~s}$ in the actual system, respectively. The results obtained for $n^{2}=2$ agree well with the numerical data for lower spatial resolutions ${ }^{(23),(24)}$. It is also confirmed that for both the values of $n^{2}$, the interface retains its initial thickness during the collapse. Figure 3 shows the time series of dimensionless horizontal and vertical positions of the contact lines $X^{*}=X / a$ at $t_{1}{ }^{*}$ and $Z^{*}=Z / H$ at $t_{2}{ }^{*}=t_{1}{ }^{*} / n$. In the figure, the solid lines denote the present results, and the circles denote the results of the experiment ${ }^{(27)}$. The numerical results for both the values of $n^{2}=1$ and 2 agree with the experimental results up to $t_{1}{ }^{*}$ and $t_{2}{ }^{*}=3.0$, respectively. As shown in Fig.4, the prediction of dimensionless velocities of the moving contact lines, $U^{*}=$ $d X^{*} / d t_{1}{ }^{*}$ and $V^{*}=d Z^{*} / d t_{2}{ }^{*}$, also agrees with the experimental data ${ }^{(27)}$.
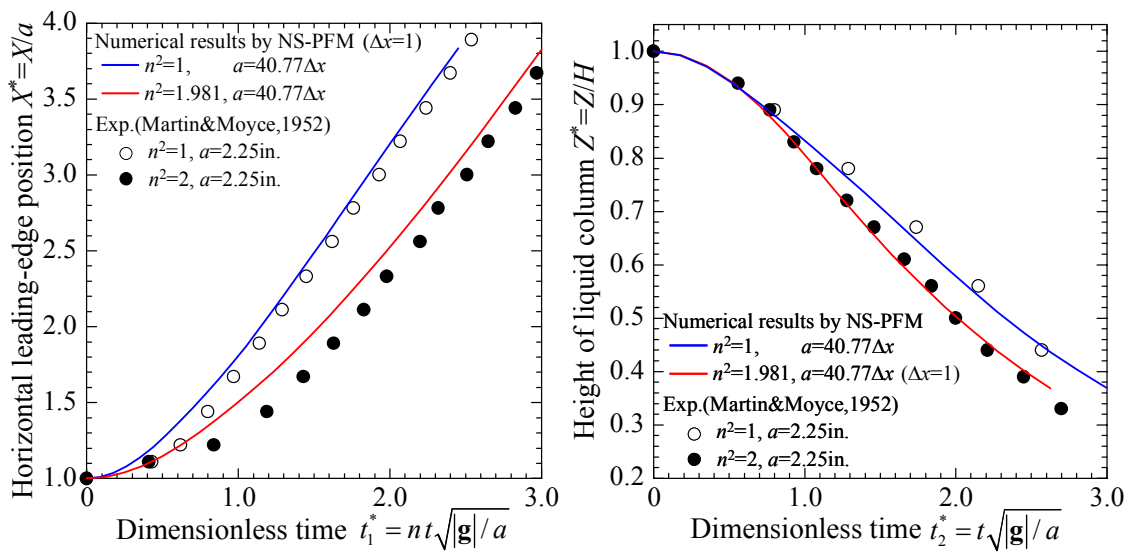

Fig. 3 Positions of the contact lines on the horizontal and vertical solid walls.
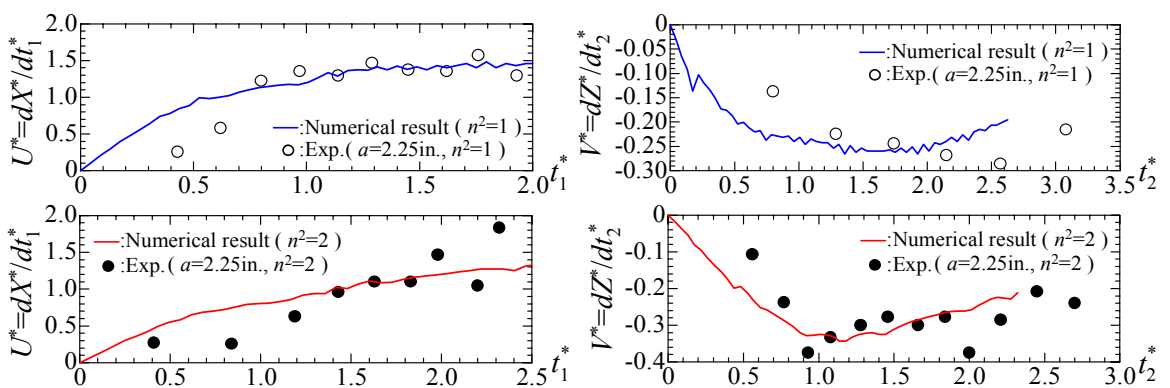

Fig. 4 Velocities of the contact lines moving on the horizontal and vertical solid walls. 


\subsection{Static contact angle}

In order to examine the validity of the wetting boundary condition, we conducted a numerical simulation of the formation of a single drop in a stagnant gas on a flat surface of a stationary solid wall ${ }^{(22)}$. The surface tension $\sigma=7.42 \times 10^{-3}$ was obtained from $\kappa_{S}=2.94$ $\times 10^{-2}$. The densities $\rho_{G}$ and $\rho_{L}$ were $1.247 \times 10^{-3}$ and 1.0 respectively; both the viscosities $\mu_{L}$ and $\mu_{G}$ were set to the value 0.01 . Initially, the shape of the drop was that of a hemisphere with a radius of approximately 15 unit cubic cells. These values lead to the dimensionless parameter $O h=3 \times 10^{-2}$. Figure 5 shows the results of the steady-state interfacial shape of the drop. As shown in Fig.6, the static contact angle $\theta_{W}$ is increased from $49^{\circ}$ to $134^{\circ}$ for $-1.5 \times 10^{-3} \leq \gamma_{S} \leq+1.5 \times 10^{-3}$.

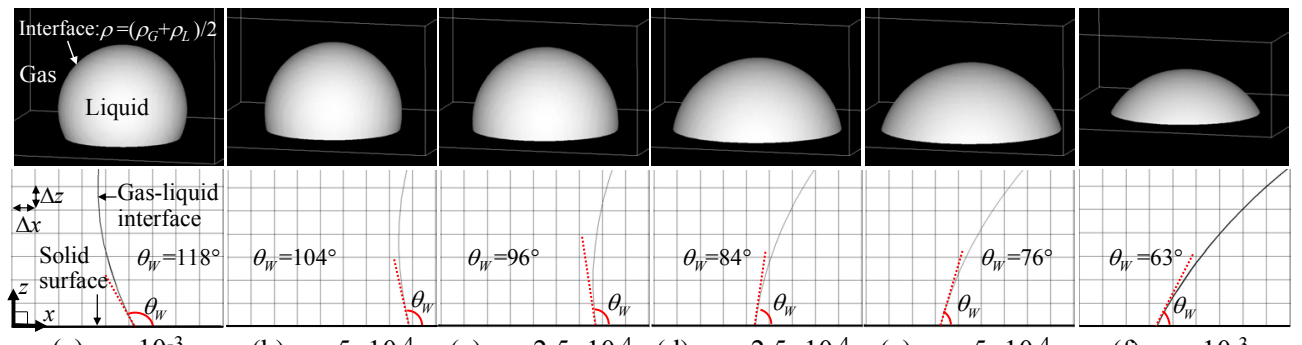

(a) $\gamma_{S}=10^{-3}$

(b) $\gamma_{S}=5 \times 10^{-4}$

(c) $\gamma_{S}=2.5 \times 10^{-4}$

(d) $\gamma_{S}=-2.5 \times 10^{-4}$

(e) $\gamma_{S}=-5 \times 10^{-4}$

(f) $\gamma_{S}=-10^{-3}$

Fig. 5 Shape of the drop on a flat solid surface under no gravity, and the static contact angle.

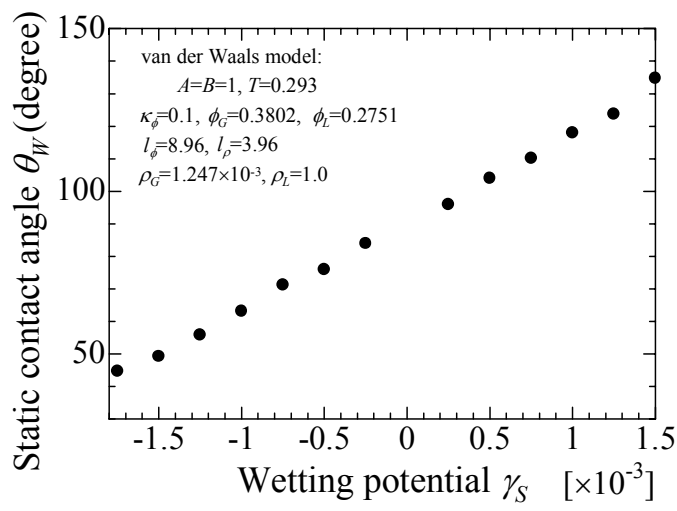

Fig. 6 Static contact angle for wetting-potential parameter $\gamma_{s}$.

\subsection{Benchmark tests for capillary force}

After the investigation of the relationship between the contact angle $\theta_{W}$ and the parameter $\gamma_{S}$, the capillary force was examined by conducting two benchmark tests for static two-phase fluid problems under gravity g, as shown in Fig.7. The first test was for the formation of a two-dimensional liquid column between parallel vertical plates (Fig.7 (a)). The gas and liquid phases were assumed to be air and water under normal gravity, respectively. The width $d$ of 32 cells was equivalent to $5 \mathrm{~mm}$ in the actual fluid system. The depth $D$ was set to be $d / 2$. The test was conducted by using the following parameter values: $|\mathbf{g}|=2 \times 10^{-3}, \mu_{G}=1.30 \times 10^{-4}, \mu_{L}=9.56 \times 10^{-3}, \rho_{G}=1.247 \times 10^{-3}, \rho_{L}=1.0$, and $\sigma=0.620$. The second test was for the deformation of a three-dimensional drop on a horizontal flat solid wall (Fig.7 (b)). This test was conducted for $|\mathbf{g}|=10^{-4}$ under the same conditions of density, viscosity, and surface tension in the simulation under no $\mathbf{g}$, which is mentioned in Sec.3.2. The shape of the drop was initially that of a hemisphere with a radius $R$ equivalent to 20 cells. In each test, the solid surface was hydrophilic with $\theta_{W}<90^{\circ}$.

The numerical results are shown in Fig.8. The theoretical prediction for the height $h$ of the liquid column in Fig.8 (a) is given by $2 \sigma \cos \theta_{W} /\left\{\left(\rho_{L}-\rho_{G}\right)|\mathbf{g}| d\right\}$, whereas that for the deformed drop is given by $2 k^{-1} \sin \left(\theta_{W} / 2\right)$ for $R>k^{-1}=\left\{\sigma /\left(\rho_{L}|\mathbf{g}|\right)\right\}^{0.5}$ (capillary length) in Fig. 8 (b). Both of the figures show good agreements between the numerical results (open 
circle) and the theoretical results (solid line); this indicates that not only the capillary force is appropriately evaluated but also the angle $\theta_{W}$ is measured with sufficient accuracy.

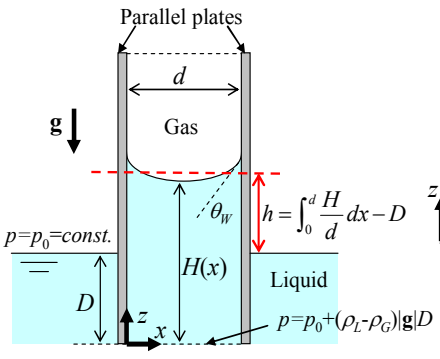

(a) 2D liquid column

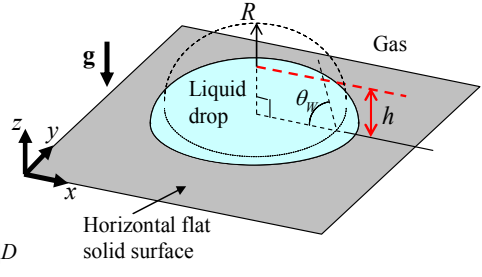

(b) 3D liquid drop

Fig. 7 Static contact line problems under gravity $\mathbf{g}$ in two and three dimensions.

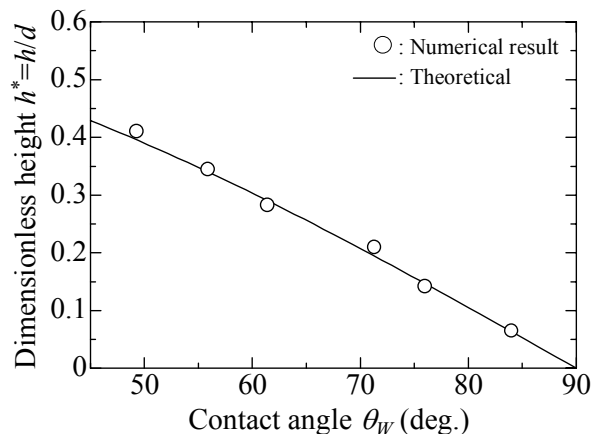

(a) 2D liquid column between parallel plates

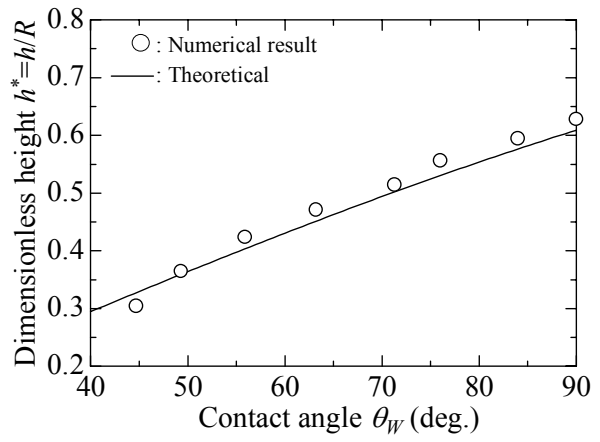

(b) 3D liquid drop on flat solid surface

Fig. 8 Numerical results of the static contact line problems under gravity in two and three dimensions.

\subsection{Two-phase flows in a rectilinear channel}

The NS-PFM was applied to the numerical simulation of motions of a two-phase fluid in a rectilinear channel with a square cross-section ${ }^{(22)}$. The fluid was assumed to be an air-water system with density and viscosity ratios $\rho_{L} / \rho_{G}=828$ and $\mu_{L} / \mu_{G}=55$, respectively, in a microchannel with sides of $100 \mu \mathrm{m}$; for numerical stability at $\Delta t=2.5 \times 10^{-3}$ or $5 \times 10^{-3}$, the effect of $\sigma$ was set as $10^{-5}$ or $10^{-3}$ times smaller than that of the actual fluid. The three-dimensional computational domain of $70 \times 20 \times 20$ unit cubic cells was surrounded by hydrophobic flat solid walls, liquid inflow boundary, and free outflow boundary under constant pressure. The liquid with $\rho_{L}=1$ was injected into the channel under conditions of constant pressure gradient $\partial p / \partial x=-3.276 \times 10^{-4}$. On the bottom wall surface, a hydrophilic region with width $a=8$ was located away from the initial position of the interface. The static contact angles $\theta_{W}=119^{\circ}$ and $61^{\circ}$ were set with $\gamma_{S}= \pm 1.044 \times 10^{-3}$, respectively.

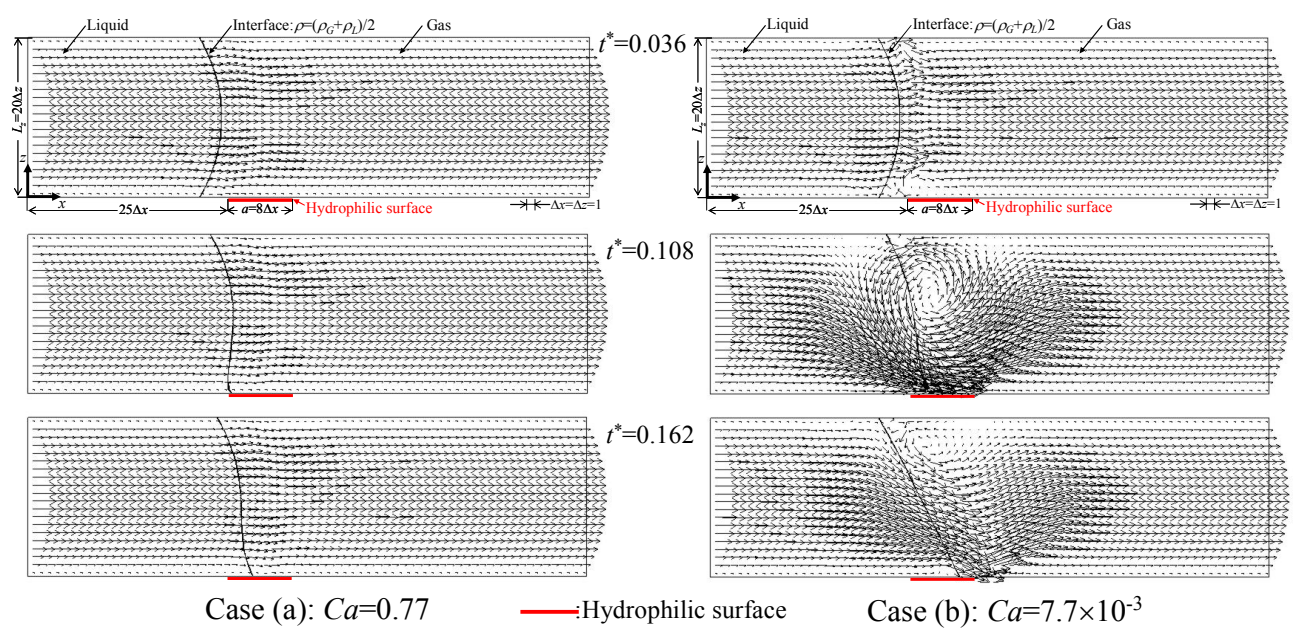

Fig. 9 Velocity distributions of the two-phase flow in a rectilinear channel 
The numerical results are shown in Figs. 9 and 10. The capillary number, $C a$, is 0.77 in Case (a) and $7.7 \times 10^{-3}$ in Case (b) for $\sigma=5.95 \times 10^{-3}$ and $5.95 \times 10^{-1}$ respectively, for the averaged inflow velocity of $\left|\mathbf{U}_{\text {in }}\right|_{\text {avg }}=3.6 \times 10^{-3}$. The Reynolds number $R e$ is 0.56 in both the cases. As shown in Fig.9, the leading edge of the liquid in Case(a) moves gradually on the hydrophilic region as compared to that in Case (b) for smaller $\mathrm{Ca}$. In the latter case, the local counter-clockwise circulation flow is caused around the interface at a dimensionless time $t^{*}=t\left|\mathbf{U}_{\text {in }}\right|_{\text {avg }} / L_{Z}=0.108$ immediately after the leading edge starts moving on the hydrophilic region. In contrast, such a circulation flow is not induced in Case (a) for larger $\mathrm{Ca}$, because the forces due to the pressure gradient and the viscous stress are dominant over the surface tension force. As shown in Fig.10, the fluid motion for smaller $\mathrm{Ca}$ is accelerated in the entire flow domain by the local effect of the capillary force on the interface, which is greater than the effect of larger values of $C a$. The result of the acceleration of the two-phase flow agrees qualitatively with the experimental data obtained in the air-water system for $\mathrm{Ca}$ $=2.7 \times 10^{-6(28)}$.
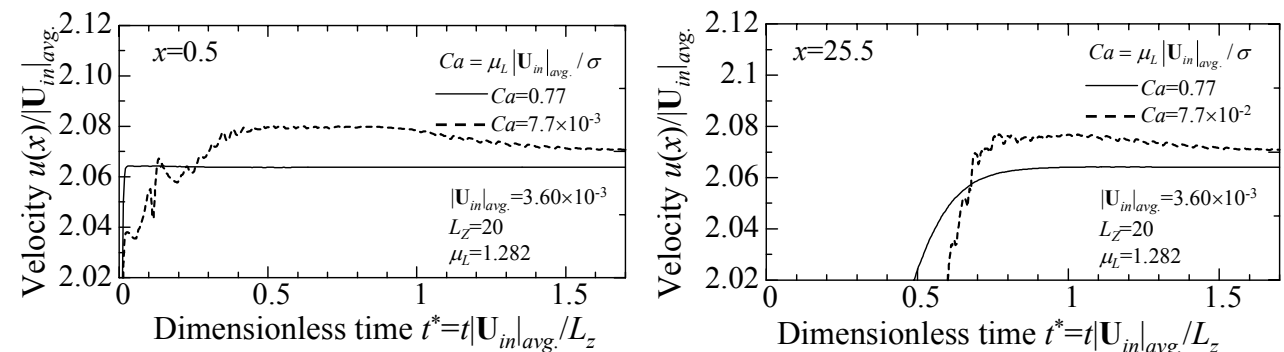

Fig. 10 Time series variation of the velocity component $u(x)$ along the $x$ direction on a center line of the channel.

\subsection{Motions of drop on solid surface with heterogeneous wettability}

The final application of the NS-PFM is to a series of three-dimensional displacement and breakup motions of a single liquid drop on a flat solid surface in a stagnant gas under no gravity ${ }^{(22)}$. This simulation was conducted for preliminarily examining the fundamental capability of the NS-PFM to predict the motions of fluid particles in micro devices that control them at high frequency by using electro-wetting-on-dielectrode (EWOD) technology ${ }^{(2)}$.
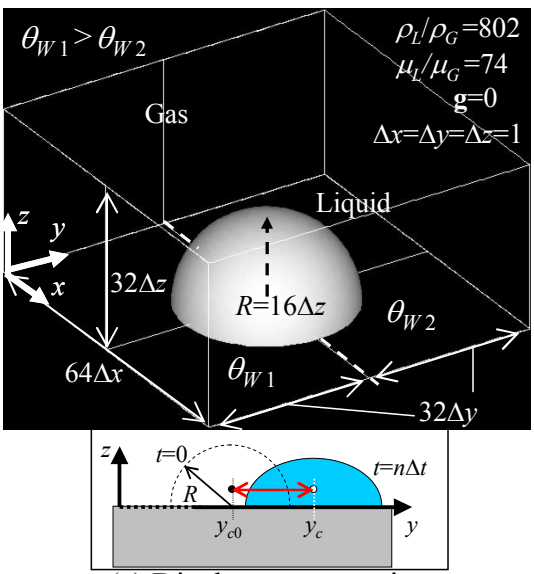

(a) Displacement motion

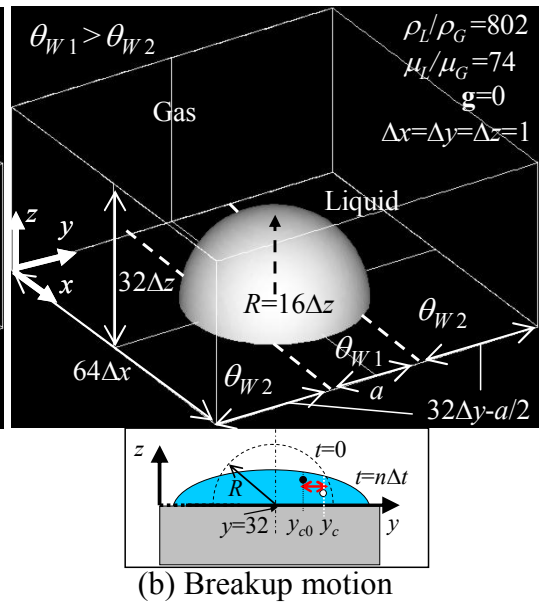

(b) Breakup motion

Fig. 11 Computational domains for the simulations of motion of a single drop in gas on solid wall.

The computational domain was discretized uniformly with $64 \times 64 \times 32$ cells and surrounded with stationary flat solid walls. On the bottom wall, a liquid drop with $\rho_{L}=1$ and $\mu_{L}=1.67 \times 10^{-3}$ was initially formed with a hemisphere shape with radius equivalent to $R=16$ cells in the gas with $\rho_{G}=1.247 \times 10^{-3}$ and $\mu_{G}=2.26 \times 10^{-5}$. The drop motions were simulated under several conditions of difference between static contact angles, $\Delta \theta_{W}=$ 
$\theta_{W 1}-\theta_{W 2}>0$, and Ohnesorge number $O h$. The angle difference $\Delta \theta_{W}$ was set as either $29^{\circ}$, $58^{\circ}, 68^{\circ}$, or $86^{\circ}$ by using a pair of values of $\gamma_{S}=0, \pm 1.044 \times 10^{-3}, \pm 1.25 \times 10^{-3}$ and $\pm 1.50 \times 10^{-3}$. The dimensionless values of $O h=1.2 \times 10^{-3}, 1.7 \times 10^{-3}, 2.4 \times 10^{-3}$ and $3.39 \times 10^{-3}$ were given by $\sigma=1.21 \times 10^{-1}, 6.06 \times 10^{-2}, 3.03 \times 10^{-2}$ and $1.51 \times 10^{-2}$, respectively. In the simulations of the displacement, the center of mass of the drop was initially positioned exactly on the border line between the hydrophilic and hydrophobic surfaces of the bottom wall (Fig.11 (a)). In the simulations of the breakup, the hydrophilic regions were located on both sides of the hydrophobic region with width $a$ on the central part of the bottom surface (Fig.11 (b)).

Table 1 Parameters in the displacement simulation.

\begin{tabular}{|c|c|c|c|}
\hline Case No. & $\gamma_{S}$ & $\Delta \theta_{W}$ (degree) & \multirow{O}{O}{ Oh $\left(\times 10^{-3}\right)$} \\
\hline 1 & $\pm 1.044 \times 10^{-3}$ & 58 & \multirow{2}{*}{1.70} \\
\cline { 1 - 3 } 2 & $\pm 1.250 \times 10^{-3}$ & 68 & \\
\hline 3 & $\pm 1.50 \times 10^{-3}$ & 86 & \\
\cline { 1 - 1 } 4 & \multirow{2}{*}{ $\pm 1.044 \times 10^{-3}$} & 58 & 2.40 \\
\cline { 1 - 1 } 5 & & & 1.20 \\
\hline
\end{tabular}

Figure 12 shows the time series variation in the drop shape, the interfacial profile and the flow velocity distribution on a $y$ - $z$ cross-section at $x=32$ in the simulations of the displacement for $O h=1.7 \times 10^{-3}$ at $\Delta \theta_{W}=58^{\circ}$ and $86^{\circ}$ in Case 1 and Case 3 (see Table 1), respectively. In both the cases, the fluid particle is assumed to be a water drop with $R=8$ $\mathrm{mm}$. The drop moves onto the hydrophilic region and out of the hydrophobic region due to the difference in capillary force. For larger $\Delta \theta_{W}$, the motion becomes rapid and then the induced flow velocity increases because the capillary force in the $y$ direction increases.
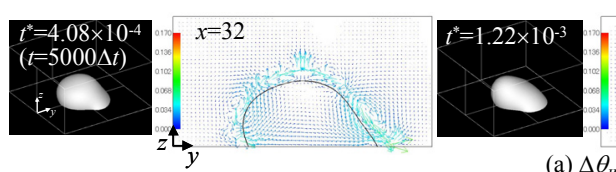

(a) $\Delta \theta_{W}=58^{\circ}$ (Case1)
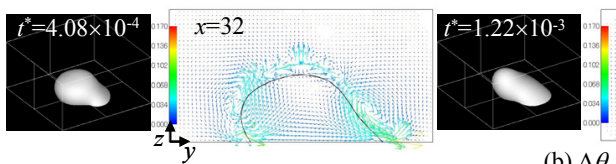

(b) $\Delta \theta_{W}=86^{\circ}$ (Case3)
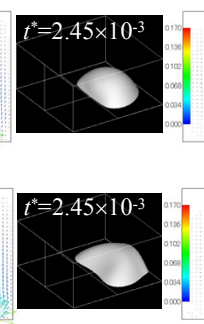

Fig. 12 Shape of the sliding drop and the velocity distribution at dimensionless time $t^{*}$ for $O h=1.7 \times 10^{-3}$.
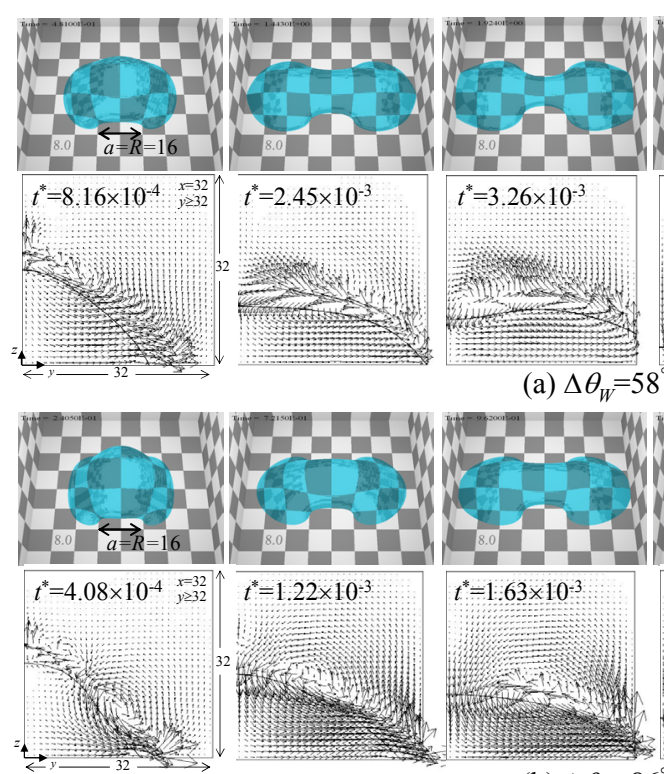

(b) $\Delta \theta_{W}=86^{\circ}$ (Case 8$)$

(a) $\Delta \theta_{W}=58^{\circ}$ (Case6)
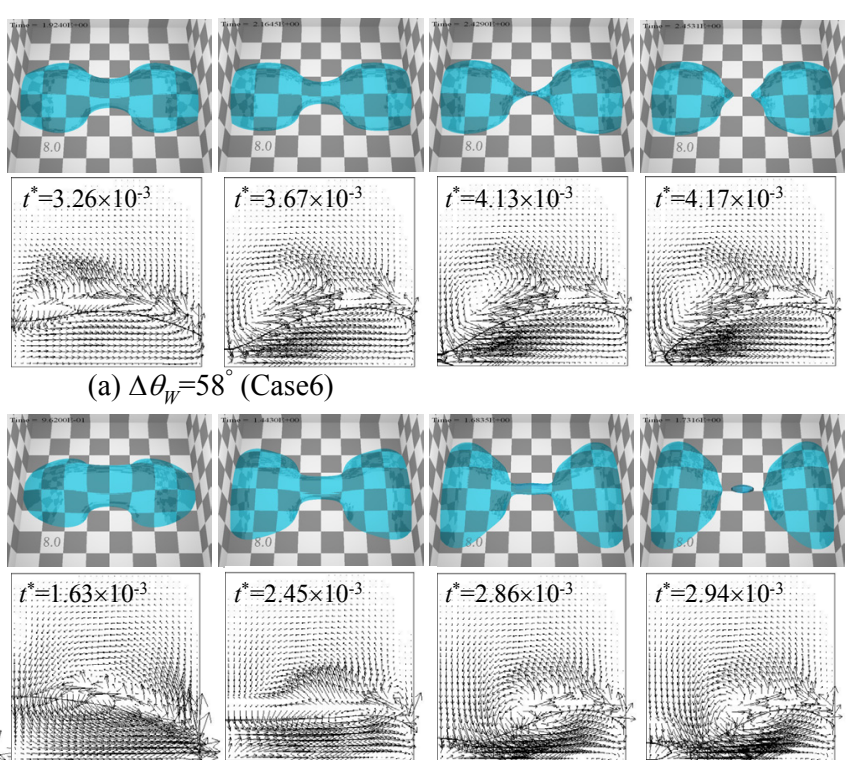

Fig. 13 Shape of the drop and the velocity distribution on a cross-section at dimensionless time $t^{*}$ in the simulations of the breakup for contact angle difference $\Delta \theta_{W}$ and $O h=1.7 \times 10^{-3}$. 
Figure 13 shows the shape of the drop and the flow velocity distribution on the vertical cross-section at each dimensionless time $t^{*}$ in the simulations of the breakup for $O h=1.7 \times$ $10^{-3}, a=R$, and $\Delta \theta_{W}=58^{\circ}, 86^{\circ}$. In both the cases, it is again assumed that a water drop in air has $R=8 \mathrm{~mm}$. The drop breaks up into a pair of twin drops at $t^{*}=4.17 \times 10^{-3}$ in the case of smaller $\Delta \theta_{W}$; however, for larger $\Delta \theta_{W}$, a tiny satellite drop is also generated between the twin drops at the earlier time $t^{*}=2.94 \times 10^{-3}$, from a throat part of the parent drop that is deformed to have a dumbbell-like shape. As shown in Table 2, the breakup occurs except for $\Delta \theta_{W}=29^{\circ}($ Case 9 and Case 10) and for $a=R / 2$ (Case 12).

Figure 14 shows the time histories of the distance of horizontal movement of the drop from its initial position on a dimensionless scale $t^{*}=t \mu_{L} \rho_{L}^{-1} R^{-2}=\left(\sigma \rho_{L}^{-1} R^{-3}\right)^{0.5} \mathrm{Oh}$. In each simulation of displacement and breakup, the distance is measured with respect to the centre-of-mass position of the entire or half body of the drop, as shown in Fig.11. The drop motion is accelerated not only as increasing values of $\Delta \theta_{W}$ but also as decreasing values of $O h$ (for larger $\sigma$ ). For all the breakup cases, the drop moves over the dimensionless distance 0.4 from its initial position.

The above results of the drop motions driven by the difference in capillary force are well predicted qualitatively in comparison with the experimental observation of a water drop in a microchannel ${ }^{(2)}$ and other numerical results ${ }^{(2),(21)}$.

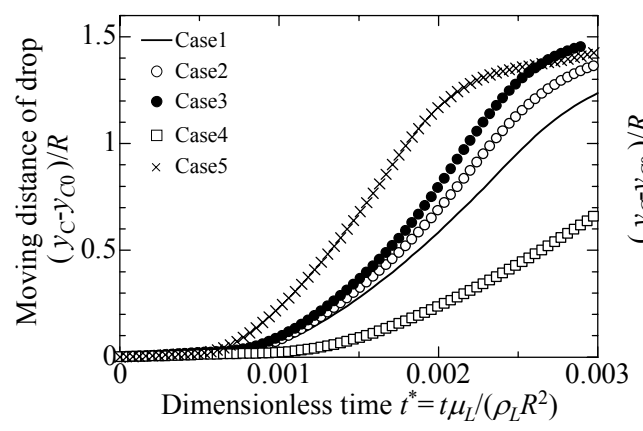

(a) Displacement motion

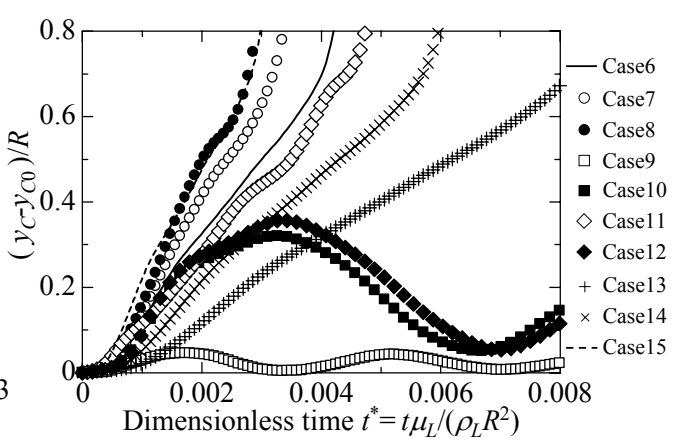

(b) Breakup motion

Fig. 14 Time histories of the distance of movement of the drop on a dimensionless time scale $t^{*}$.

Table 2 Parameters and results in the simulations of the breakup (Y: breakup, N: no breakup)

\begin{tabular}{|c|c|c|c|c|c|c|c|c|c|}
\hline Case No. & $\Delta \theta_{W}$ (degree) & $a^{*}=a / R$ & $O h\left(\times 10^{-3}\right)$ & Break & Case No. & $\Delta \theta_{W}$ (degree) & $a^{*}=a / R$ & Oh $\left(\times 10^{-3}\right)$ & Break \\
\hline 6 & 58 & \multirow{5}{*}{1} & \multirow{5}{*}{1.70} & $\mathrm{Y}$ & 11 & \multirow{5}{*}{58} & 1.5 & \multirow{2}{*}{1.70} & $\mathrm{Y}$ \\
\hline 7 & 68 & & & $\mathrm{Y}$ & 12 & & 0.5 & & $\mathrm{~N}$ \\
\hline 8 & 86 & & & Y & 13 & & & 3.39 & Y \\
\hline 9 & $29\left(\theta_{W 2}=90\right)$ & & & $\mathrm{N}$ & 14 & & 1 & 2.40 & $\mathrm{Y}$ \\
\hline 10 & $29\left(\theta_{W 1}=90\right)$ & & & $\mathrm{N}$ & 15 & & & 1.20 & $\mathrm{Y}$ \\
\hline
\end{tabular}

\section{Conclusions}

In this study, one of phase-field methods ${ }^{(11),(24)}$, NS-PFM ${ }^{(7),(22)-(24)}$, was applied to moving contact-line problems for examining its fundamental capability for simulating the motions of a two-phase fluid with high density ratio on a solid surface. This method can be used to solve a set of Navier-Stokes and interface-advection equations combined with the diffuse-interface model based on the free-energy theory ${ }^{(10),(11)}$, without using conventional elaborating interface-tracking algorithms ${ }^{(12)}$. A simple wetting boundary condition on the solid surface ${ }^{(22)}$ was adopted based on the free-energy approach ${ }^{(4)-(6)}$.

The following major findings were obtained through $2 \mathrm{D}$ and $3 \mathrm{D}$ simulations at a density ratio of approximately 800: (1) the contact line motion of the 2D liquid column on flat solid wall under gravity was predicted and observed to be in good agreement with the experimental data ${ }^{(27)}$; (2) the static contact angle of the liquid drop on a solid surface varies 
almost linearly with the value of the wetting potential parameter; (3) the capillary force was evaluated with sufficient accuracy in the static contact line problems on the liquid column and drop under gravity; (4) the interfacial motion of the two-phase fluid in a rectilinear channel is accelerated on a hydrophilic region of the solid surface; (5) the motion of the fluid in the channel was accelerated in the entire flow domain for larger surface tension. This agreed qualitatively with the experimental data ${ }^{(28)}$; and (6) the NS-PFM successfully captured the displacement and breakup motions of a single drop caused by the difference in capillary force on the flat solid surface. The results were well predicted qualitatively in comparison with the available data ${ }^{(2),(21)}$.

The numerical results provide proof to support that the NS-PFM that includes the boundary condition can be employed for simulating capillarity-driven two-phase fluid motions on a surface with heterogeneous wettability for high-density ratios equivalent to that of an air-water system. However, the applicability of this method to practical problems must be examined more qualitatively and quantitatively in comparison with other data. One of our future aims is to reduce the parasitic current around the interface that occurs due to numerical error in the surface-force balance, because such an error current would hide the physical flow in the microscopic field where the surface tension force is dominant. This can be achieved by adopting a higher-order finite difference scheme or an energy-conserving discretization technique ${ }^{(13)}$ to solve the NS equations. After the improvement, the NS-PFM will be more applicable to the simulations of a two-phase flow in microdevices ${ }^{(1)-(3),(28)}$. Moreover, the numerical results by the method will be compared in more detail with those by the two-phase LBMs ${ }^{(18),(21)}$ and other methods in future work.

\section{Acknowledgements}

This study was supported by the Japan Ministry of Education, Culture, Sports, Science and Technology (MEXT) and the Japan Society for the Promotion of Science (JSPS) under the Grant-in-Aid for Scientific Research (No.18760134).

\section{References}

(1) Ichikawa, N., Hosokwa, K. and Maeda, R., Interface motion of capillary-driven flow in rectilinear microchannel, J. Colloid Interface Sci., Vol.280 (2004), pp.155-164.

(2) Berthier, J., Clementz, Ph., Raccurt, O., Jary, D., Claustre, P., Peponnet, C. and Fouillet, Y., Computer aided design of an EWOD microdevice, Sens. Actuator A - Phys., Vol.127 (2006), pp.283-294.

(3) Inoue, G., Yoshimoto, T., Matsukuma, Y. and Minemoto, M., Development of simulated gas diffusion layer of polymer electrolyte fuel cells and evaluation of its structure, J. Power Sources, Vol.175 (2008), pp.145-158.

(4) Seppecher, P., Moving contact lines in the Cahn-Hilliard theory, Int. J. Eng. Sci., Vol.34 (1996), pp.977-992.

(5) Jacqmin, D., Contact-line dynamics of a diffuse fluid interface, J. Fluid Mech., Vol.402 (2000), pp.57-88.

(6) Briant, A. J. ,Papatzacos, P. and Yeomans, J. M., Lattice Boltzmann simulations of contact line motion in a liquid-gas system, Phil. Trans. R. Soc. Lond. A, Vol.360 (2002), pp.485495.

(7) Takada, N., and Tomiyama, A., A numerical method for two-phase flow based on a phase-field model, JSME Int. J. Ser. B - Fluids Therm. Eng., Vol.49 (2006), pp.636-644.

(8) Nadiga, B. T. and Zaleski, S., Investigations of a two-phase fluid model, Eur. J. Mech. B Fluids, Vol.15 (1996), pp.885-896.

(9) Anderson, D. M., McFadden, G. B. and Wheeler, A. A., Diffuse-interface methods in fluid mechanics, Annu. Rev. Fluid Mech., Vol.30 (1998), pp.139-165.

(10) van der Waals, J. E., The thermodynamic theory of capillarity under the hypothesis of a 
continuous density variation, Transl. Rowlinson, J. S., J. Stat. Phys., Vol.20 (1979), pp.197 -244 .

(11) Cahn, J. W. and Hilliard, J. E., Free energy of a nonuniform system. I. Interfacial free energy, J. Chem. Phys., Vol.28 (1958), pp.258-267.

(12) Kothe, D. B., Perspective on Eulerian finite volume methods for incompressible interfacial flows in H. C. Kuhlmann, H. -J. Rath eds. Free Surface Flows, Springer Verlag WIEN, Austria (1998), p.267.

(13) Jamet, D., Torres, D. and Brackbill, J. U., On the theory and computation of surface tension: the elimination of parasitic currents through energy conservation in the second-gradient method, J. Comput. Phys., Vol.182 (2002), pp.262-276.

(14) Badalassi, V. E., Ceniceros, H. D., Banerjee, S., Computation of multiphase systems with phase field model, J. Comput. Phys., Vol.190 (2003), pp.371-397.

(15) Kim, J., A continuous surface tension force formulation for diffuse-interface models, J. Comput. Phys., Vol.204 (2005), pp.784-804.

(16) Swift, M. R., Orlandini, E., Osborn, W. R. and Yeomans, J. M., Lattice Bolztammn simulations of liquid-gas and binary fluid systems, Phys. Rev. E, Vol.54 (1996), pp.50415052.

(17) Teng, S., Chen, Y. and Ohashi, H., Lattice Boltzmann simulation of multiphase fluid flows through the total variation diminishing with artificial compression scheme, Int. J. Heat Fluid Flow, Vol.21 (2000), pp.112-121.

(18) Inamuro, T., Ogata, T., Tajima, S. and Konishi, N., A lattice Boltzmann method for incompressible two-phase flows with large density differences, J. Comput. Phys., Vol.198 (2004), pp.628-644.

(19) Seta, T. and Okui, K., The single component thermal lattice Boltzmann simulation of pool boiling in two dimensions, Journal of Thermal Science and Technology, Vol.1 (2006), pp.125-137.

(20) Yoshino, M. and Mizutani, Y., Lattice Boltzmann simulation of liquid-gas flows through solid bodies in a square duct, Math. Comput. Simul., Vol.72 (2006), pp.264-269.

(21) Yan, Y. Y. and Zu, Y. Q., A lattice Boltzmann method for incompressible two-phase flows on partial wetting surface with large density ratio, J. Comput. Phys., Vol.227 (2007), pp.763-775.

(22) Takada, N. and Tomiyama, A., Numerical simulation of isothermal and thermal two-phase flows using phase-field modeling, Int. J. Mod. Phys. C, Vol.18 (2007), pp.536-545.

(23) Takada, N., Misawa, M. and Tomiyama, A., A phase-field method for interface-tracking simulation of two-phase flows, Math. Comput. Simul., Vol.72 (2006), pp.220-226.

(24) Takada, N. and Tomiyama, A., Application of interface-tracking method based on phase-field model to numerical analysis of free surface flow, Theoretical and Applied Mechanics Japan, Vol.55 (2006), pp.149-156.

(25) Chorin, A. J., Numerical solution of the Navier-Stokes equations, Math. Comput., Vol.22 (1968), pp.745-762.

(26) Kawamura, T. and Kuwahara, K., Computation of high Reynolds number flow around a circular cylinder with surface roughness, AIAA Paper, No.84-0340 (1984).

(27) Martin, J. C. and Moyce, W. J., An experimental study of the collapse of liquid columns on a rigid horizontal plane, Philos. Trans. Roy. Soc. London, Ser. A, Vol.244 (1952), pp.312324.

(28) Ichikawa, N., Hosokawa, K., Maeda, R. and Ichiki, M., PIV measurements using high speed camera for instantaneous liquid penetration with wetted spot and trapped liquid drop at corners in micro-channels, Proceedings of 10th International Symposium on Flow Visualization (2002), CD-ROM, Paper No.F0253. 